This forum is dedicated to personal health in all its many facets: decision-making, goal setting, celebration, discovery, reflection, and coordination, among others. We look at innovations in interactive technologies and how they help address current critical healthcare challenges. - Gillian R. Hayes, Editor

\title{
mHealth + Proactive Well-being $=$ Wellth Creation
}

Elizabeth F. Churchill, eBay, m.c. schraefel, University of Southampton

s of late 2013,39
percent of the
world's population
is connected to the
Internet. Europe
stands at 68.6 percent,
with the EU at 76.5

percent; the U.S. is at 84.9 percent, with all the Americas at 62.3 percent. Even areas known to have a higher percentage of their population in the lower portion of the economic pyramid are showing steady increases in people accessing the Internet: Africa is at 21.3 percent and rising rapidly, while India is at 15.8 percent [1].

It is clear that advances in the design of ICTs are transforming health practices, from bedside patient support to remote patient outreach, the latter having a special focus and success rate in the developing world (e.g., [2]). mHealth, the practice of medicine and public health supported by mobile devices, is a major focus of investment for governments, organizations, and businesses. In 2013, a number of states in the U.S. began rolling out mobile applications to alert residents of health issues, promote exercise-related events, and encourage engagement in proactive healthcare activities [3]. While most activities target individuals, clearly there are broader family and community benefits.

There are also workplace and business benefits. Healthier individuals are more engaged in their work and typically take less time off [4]. Plus, a highly lucrative business has emerged for personal health and fitness devices, applications, and services. Apple's iOS now includes a health app that leverages the iPhone's motion sensor, offering basic motion counts such as daily steps taken - just one example of the emerging landscape of mHealth devices.

\section{THE DAGSTUHL PERSPECTIVES WORKSHOP}

Although much research has focused on body maintenance and repair, focusing on the achievement of fitness goals and/or following medicalized concepts of health management, there is an emerging complementary research agenda that calls for a focus on the experience of well-being and increasing quality of life. This latter research agenda focuses on the phenomenological aspects of wellbeing, broadening the discourse beyond health as the absence of illness and healthcare as disease and/or condition prevention/management (see, for example, [5]). This is what leads us to the idea of "wellth creation,"

\footnotetext{
Insights

$\rightarrow$ Health is more than illness prevention. "Wellth creation" is the development of sustained strategies for increasing the experience of well-being.

$\rightarrow$ Proactive health and well-being means culturally constructing "a better normal."

$\rightarrow$ Wellth creation will involve rethinking: notions of health, interactive technology design, the form and role of health-related data, and engagement with stakeholder groups.
}

an intentional play on words to underscore our belief that personal well-being or "wellth" offers broader societal gains.

This shift in perspective from the body as maintained, repaired, and/or mechanistically managed to designing for experienced well-being invites (re)design thinking. We recently organized a workshop at Schloss Dagstuhl [6] to create a companion agenda to technologically managed healthcare: technologically supported proactive well-being. Participants at the workshop hailed from HCI but also from other disciplines, including medicine, sports science, neuroscience, psychology, sociology, and computer science. This domain diversity reflects not only the current multidisciplinarity of HCI but also new needs and domain expertise beyond existing HCI practice. At the workshop we outlined four areas for foundational research. We address each of these areas in turn.

\section{Rethinking epistemological} foundations in the health app space. Mobile phones, sensors, tracking devices, social media technologies, and emerging techniques to capture, store, manage, and visualize data are front and center in the emerging “health app" landscape. However, too often the dominant rhetoric for both the healthcare domain and the world of fitness applications draws from the medical model of measurement and management that applies quantized norms and benchmarks to functions of the human body. From this epistemological foundation and underlying logic, the motivational 
drivers for most fitness or healthcare initiatives are as follows:

- Self-care in the form of monitoring and feedback will lead to healthier individuals.

- By engaging in self-measurement and (potentially collaborative) monitoring and behavior adjustment/ management, individuals will proactively prevent health issues.

- By engaging in such activities, people will be better able to manage existing conditions and will participate in reducing the burden on healthcare professionals and on the healthcare system as a whole, and on their informal caregivers (family, friends, community).

The Perspectives Workshop started with debating what would constitute a broader perspective than illness prevention for ICTs in the healthcare space, acknowledging that overall mental and physical well-being are of central import not just for preventing illness in the first place, but also for the management of acute and chronic ailments. We asked: What are contemporary ways of knowing and doing in this space, and what opportunities are there for new approaches? Plenty of research and practice informs us about what specific activities can be "good for us." However, how do we conceptualize, represent, and then present activities in ways that "work for me in my life"? Or, more fundamentally, is a specific-activity focus the right one? If not that form of intervention, then what should intervention be? Where do we get our ideas of what a "normal" body is? How can we rethink "normal" benchmarks to reflect more personal and individual ideas of "normal for me"? A new "normal" that includes an experience of well-being is needed.

Technology and design. Behaviorchange interventions typically target the conscious self, appealing to us as rational beings: It's good for us, therefore we will of course integrate well-being into our lives. But it is also essential to highlight the role of the senses and emotions as we consider new sociotechnical design paradigms. Humans are naturally inclined to make choices that are instantaneously rewarding - the release of certain bodily chemicals such as endorphins is

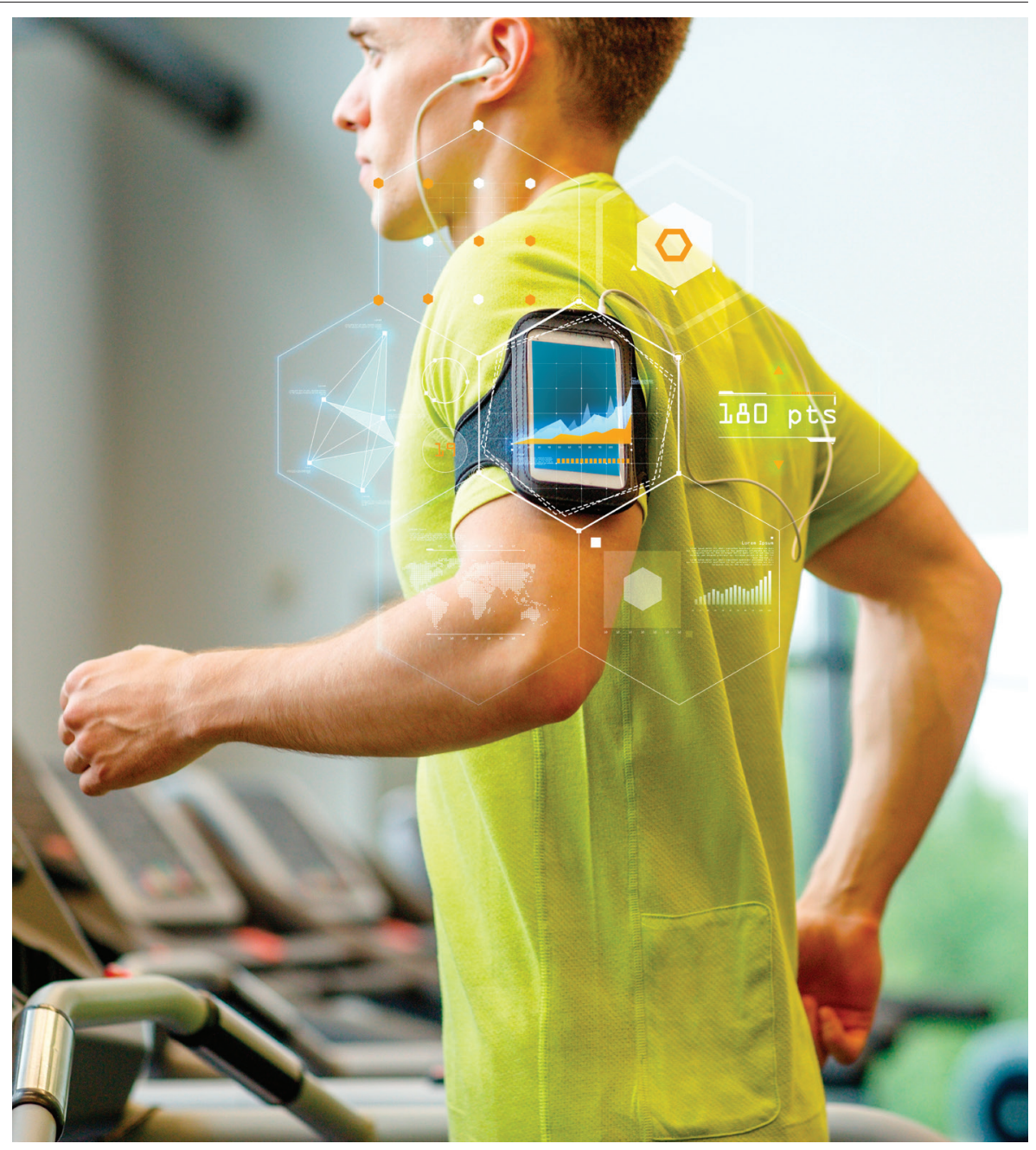

pleasant - rather than rewarding in the long-term. Can we design technologies that filter or augment our perception of the world (utilizing augmented reality or brain-computer interfaces, for example) to make well-being more appealing at an emotional, visceral level? Much research can be done to create models of motivation beyond the conscious and rational, toward models that acknowledge the impact of unconscious processing. Increasingly, we can design technologies that work with unconscious processing (e.g., training aids while sleeping) and/or are rooted in autonomous actions (e.g., fight or flight).

As an illustration of the difference between fitness/healthcare and wellbeing, we know that many people engage with fitness trackers and monitors with good intentions but abandon them or feel oppressed by them. A few find pleasure or "flow" in their use, but this is not the norm. Therefore, since proactive health requires a lifelong commitment, the time dimension is critical in addressing motivation. We know that people's commitment will wax and wane over time; we need to understand how motivation operates over longer cycles, that is, how to initially involve people in proactive health and how to foster their return should their commitment wane. There is more to health than an emphasis on change. How do we design to align with people's aspirations rather than assuming participants must change? How does that framing affect our interventions, and potential participants' use of such designs?

\section{Refocusing measures and} metrics. Many inroads can be made through rethinking measurement and metrics. Data is the topic of the day, and the battleground on which personal, business, and governmental agency is currently being fought. Data is a boundary object between different facets and different stakeholders in the 


\section{DFORUM HEALTH MATTERS}

\begin{tabular}{|l|l|}
\hline \multicolumn{2}{|c|}{ Dominant rhetoric } \\
\hline Macro & Healthcare, macro-economics \\
\hline Meso & $\begin{array}{l}\text { Business logics, institutional logics, } \\
\text { legal concerns }\end{array}$ \\
\hline
\end{tabular}

legal concerns
Technology focus

Platform, infrastructures, service integration

Patient-provider communications, access to services; workplace technologies; community and social communities, including empathic communities and communities of need and interest
Representation of "users"

Data aggregates, policies, standards, guidelines

Segmented data for service "personalization" and customization, guidelines and policies
Micro Fitness, chronic and acute disease
management, fear/image driven
End-user facing devices, applications, behavior tracking and monitoring
Activity data, logging, focus on individual practice, individual level personalization

$\rightarrow$ Table 1. Macro-, meso-, and micro-level engagements with wellness and healthcare rhetorics and logics,

and their influence on technology, data, and user representations and foci.

emerging sociotechnical landscape that includes health and well-being.

However, we have much work to do in understanding the aggregation and disaggregation of data, especially when it comes to proactive well-being. When is an aggregate useful, and when is it not? Well-being is essentially and necessarily personal, reflecting the $N$ of 1 (aka $m e$ ). Health science traditionally relies on statistical information from large populations to understand the relationship between behaviors, demographics, or other kinds of antecedents and health outcomes. We then use this information to offer prescriptive or corrective advice to achieve some goal. However, these statistical tests generally offer reliable information only when there are fairly large groupings of people that are similar in a given distribution. That is, we can offer good advice to people when they fall in the "body" of the distribution - the middle 60 to 80 percent of the population for a given variable (e.g., age, race, gender, diet, exercise regimen, body type, BMI, resting heart rate, etc.). However, this fails for those in the "tails" of the distribution, those who fall on either side of the middle clump of people. It also fails when the distribution is more evenly distributed, or flat. This is even more complicated because most people fall in the middle of a distribution on some factors, but out in the tails on others - I might be very similar to most people in my need for sleep, but respond very differently from the bulk of the population to a vegetarian diet. Someone else might be like most people on both of these factors but for some reason find it very easy to maintain a reasonable BMI with very little exercise.
Health science has traditionally been very useful for people when they fall in the body of the distribution, but when they are in the tails, it can recommend advice that just doesn't work (or is even harmful).

This is where recent advances in data science can be very helpful. By exploring very large data sets of many people, signals in the tails become understandable. Machine-learning and information-visualization techniques allow us to make sense of a large number of features (different factors) and how they relate to each other to result in meaningful classifications and groupings at an individual level. The classic example of this in other domains is personalization in shopping or entertainment (Amazon, Netflix, Google, etc.). There is an opportunity to innovate in similar techniques to make huge strides in how we understand wellness information on a personal level based on the aggregate data of millions of other people providing vast amounts of individual data from new sensors and other sources.

We propose an engagement with measures. In capturing my and your data, as well as "big" and "small” data, we are particularly interested in opportunities that big my-you data collections in proactive health may afford. Further, it is crucial that we

\section{It is crucial that we overcome the artificial boundaries between qualitative and quantitative data.}

overcome the artificial boundaries between qualitative and quantitative data and realize that the quantitative measures gathered are determined by what is considered to be worth measuring or what is technologically measurable.

Stakeholders. HCI has always been concerned with systems, understanding clearly that business imperatives (i.e., "the market”), but also standards, guidelines, and policies, have an indirect but very definite effect on what can be called the direct interaction layer. Indeed, interaction programming at the device and data level is deeply constrained and/or enabled by these factors. This has been called the blunt part of HCI design, whereas the sharp part is where much interaction design focuses: the interfaces that enable interaction with services, devices, and applications [7].

While current technology design focuses on the individual, expanding and improving current models of motivation that are inscribed into interaction mechanisms, we need to acknowledge that the individual might not have schedule flexibility, making well-being a social, organizational, institutional, and perhaps even a governmental issue. From this, we perceive three broad design and data levels that correspond to different stakeholders in the arena of health and proactive well-being: macro, meso, and micro.

At the macro level, which one can consider the societal level, governments are keen to reduce the cost of healthcare provision-personal "wellth creation" 
will result in societal wealth benefits (i.e., cost savings). At the meso level exist a slew of stakeholders that include insurance agencies, healthcare providers, and social groups. We also need to consider workplaces. Well-being is often not prioritized; rather, it is subsumed and subjugated by beliefs about pressures of work and home, or constraints imposed by the infrastructure of our everyday lives. Consequently, those needing to improve well-being are the least likely to have patterns of activity and action supported by interactive tools. At the micro or individual and personal data level, we need to directly engage with people other than those already served: those who are at risk or who already suffer from conditions that require management (e.g., diabetics), fitness fanatics and athletes, and/or technology or data-obsessives who love tracking and exploring the potential of the emerging technological landscape. We need to address reasons for abandonment and find motivators and design environments structured toward reengagement.

Table 1 summarizes some of our workshop thinking on the current state of the art and where we'd like to see developments.

\section{TAKEAWAYS}

In this brief overview of an emerging area as seen through the eyes of the attendees at our Dagstuhl Seminar, we draw from and engage with a rethinking of healthcare design to include a focus on proactive well-being. A number of areas of investigation are unfolding, and we will be convening more workshops in these areas in the upcoming months and years. Our goal is to draw on ideas proposed by others in this space and to promote holistic well-being in terms of a research framework of interlocking research activities, thus complementing preventive and response-focused healthcare. We leave you with some questions to pique your interest and as an invitation to join us at future events:

- On a research level, what needs to be done to deepen our mind-body understanding - the causes, effects, mechanisms, loops, and relationships?
How might we change our conceptions of health to include concepts that foreground the experience of well-being in our complex everyday daily lives, played out over a lifetime?

- At the technology-design level, how can we embed well-being into fitness applications by design, beyond incremental physical improvements? How might we design technology that works in harmony with our less conscious brain to good effect (e.g., making fuller use of persuasive technologies, exploring new areas of influence such as during sleep, creating highly personal embedded technologies such as implants)? How can we design to rely more on sensors than manual user input (reducing the burden of active engagement)?

- At the level of motivation and personal practice, can we encourage self experiments and reflective practice? How might we quantify, qualify, share, and reflect on wellbeing success? How could we better introduce more pervasive participation in removing/reducing well-being impediments? How do we promote access to knowledge and expertise, as in the call to "bring personal training to the masses"?

-At the cultural and social level, can we design interventions to help us shift assumptions at the individual level and filter these up to create a form of "culture hack" to propagate large-scale societal change in bottom-up ways? That is, can we identify small structural changes that could have large net effects (i.e., simulating the effects and implications of micro lifestyle changes at a macro scale)? How can we use data to design for broader infrastructure changes (e.g., making the case for cultural shifts in organizations, encouraging normative shifts)?

\section{ACKNOWLEDGMENTS}

This work was completed while the Elizabeth Churchill was director of human computer interaction at eBay Research Labs in San Jose, CA. She is now director of user experience at Google. We would like to acknowledge all the attendees at the Perspectives
Workshop and the organizers and staff of Schloss Dagstuhl who supported the workshop. In particular, we would like to thank Ed Cutrell, Alan Chamberlain, Adrian Friday, and Les Nelson, who directly contributed text and/or comments on this forum article.

\section{ENDNOTES}

1. Internet World Stats: Usage and Population Statistics. (accessed Aug. 28, 2014); http:// www.internetworldstats.com/stats.htm

2. Littman-Quinn, R. et al. mHealth applications for telemedicine and public health intervention in Botswana. Proc. IST-Africa Conference. IEEE, 2011; http://ieeexplore.ieee.org/xpls/abs_all. jsp?arnumber $=6107376 \&$ tag $=1$

3. Sifferlin, A. To promote wellness, public health departments are launching apps. Will they work? Time. Aug. 30, 2013; http://healthland. time.com/2013/08/30/to-promote-wellnesspublic-health-departments-are-launchingapps-will-they-work/

4. Workplace health programs can increase productivity, Center for Disease Control and Prevention report; http://www.cdc.gov/ workplacehealthpromotion/businesscase/ benefits/productivity.html

5. Grinter, R., Siek, K., and Grimes, A. Is wellness informatics a field of human-centered health informatics? Interactions 17, 1 (2010), 76-79; http:// interactions.acm.org/archive/view/ january-february-2010/featureis-wellnessinformatics-a-field-of-human-centeredhealth-informatics

6. Dagstuhl Perspectives Workshop \#14272, Exploring Interdisciplinary Grand Challenges in ICT Design to Support Proactive Health and Wellbeing, Jun. 29-Jul. 2, 2014; http://www. dagstuhl.de/de/programm/kalender/ semhp/?semnr $=14272$

7. Reason, J. Human Error. Cambridge University Press, Cambridge, U.K., 1990.

\footnotetext{
(4) Elizabeth Churchill (axeeliz) has been working in $\mathrm{HCl}$ and user experience for 20 years. She is the executive vice president of ACM SIGCHI and a distinguished scientist of the ACM. Her co-authored volume Foundations for Designing User-Centered Systems was published in early 2014. $\rightarrow$ churchillaacm.org
}

(4) m.c.schraefel (amcphoo; http://www.ecs. soton.ac.uk) is a professor in computer science and a certified strength and conditioning coach, nutrition coach, and neural movement coach. Her work explores how we can design interactive systems to support the brain-body connection, improve performance and quality of life, and create a better normal. $\rightarrow$ mcdecs.soton.ac.uk 